Fernández-Larragueta, S., Fernández-Sierra, J. y Rodorigo, M. (2017). Expectativas socioeducativas de alumnàs inmigrantes: escuchando sus voces. Revista de Investigación Educativa, 35(2), 483-498

DOI: http://dx.doi.org/10.6018/rie.35.2.257591

\title{
Expectativas socioeducativas de alumnas inmigrantes: escuchando sus voces
}

\section{Social and educational expectations of immigrant female students: listening to their voices}

\author{
Susana Fernández-Larragueta*, Juan Fernández-Sierra* y Monia Rodorigo* \\ * Dpto Educación-Universidad de Almería-España
}

\begin{abstract}
Resumen
La incorporación de estudiantes inmigrantes en nuestras escuelas, ha generado la necesidad de construir espacios interculturales que favorezcan, además del desarrollo cognitivo, su inclusión académica y sociolaboral. En el marco de un estudio multicaso más amplio, hemos llevado a cabo doce historias singulares de vida socioeducativa a alumnas inmigrantes, que nos ha permitido conocer a través de sus voces qué elementos tienen incidencia significativa en la construcción de sus expectativas sociales y educativas. Se ha elaborado un relato cruzado a partir del análisis cualitativo de temas emergentes categorizando información de diversos instrumentos. El estudio confronta cómo ciertas creencias estereotipadas del profesorado y prácticas pedagógicas envejecidas, junto a ciertas presiones machistas y xenófobas del ambiente, producen desamparo educativo y desconfianza en dichas jóvenes. Pero también, cómo el apoyo de las madres, de compañeras y algunos docentes innovadores y comprometidos, ponen el contrapunto recreándoles ilusiones y expectativas positivas de futuro escolar y sociolaboral.

Palabras clave: expectativas; identidad; alumnas inmigrantes; inclusión
\end{abstract}

Correspondencia: Susana Fernández-Larragueta, sfernan@ual.es 


\begin{abstract}
Female immigrants incorporation in our schools has generated the need to build up intercultural spaces, promoting not only cognitive development, but also academic and social-working inclusion. In a broader multi case study's framework, a research of twelve singular stories of socio educational life -understood as personalized narrative constructions- has allowed us to know, thanks to their voices, which elements are significant to them in order to build up their social and educational expectations. Qualitative data analysis of emerging topics have been used to show how they build their future aspirations and describe them through categories. The study seeds how stereotyped teachers beliefs and traditional pedagogy, and also sexist pressure and xenophobic environments, produce educational helplessness and mistrust in those students. On the contrary, the support of mothers, partners and some innovative and committed teachers make the counterpoint, recreating positive expectations about school and labour market in the future.

Keywords: expectation; identity; foreign students; inclusion.
\end{abstract}

\title{
Introducción
}

La incorporación de alumnado inmigrante a nuestras escuelas ha supuesto un gran reto educativo que está forzando al sistema a lograr niveles de inclusión aceptables. Sin embargo, la pedagogía tradicional, preocupada en comprender y normalizar la acción didáctica centrada, básicamente, en la lógica de las disciplinas académicas y su transmisión al estudiantado, no es válida para explicar ni estimular los múltiples procesos por los que los estudiantes y, especialmente, las jóvenes inmigrantes construyen sus competencias, entendidas como la capacidad para responder a demandas complejas mediante la internalización de conocimientos, habilidades, emociones, valores y actitudes (Delors,1996; DeSeCo, 2003; Pérez Gómez, 2008; Tejada Fernández, \& Ruiz Bueno, 2016). El modelo curricular netamente disciplinar desatiende en gran parte la implicación cultural, social, profesional y de género, potenciando prácticas pedagógicas que están produciendo altos niveles de abandono escolar en nuestro país: $21.9 \%$ en España frente al 11.1\% en la Unión Europea, llegando al 41.6\% el abandono temprano del alumnado inmigrante (INE, 2015).

Evidentemente no podemos obviar, hablando de alumnado inmigrante, la incidencia del estatus laboral y socioeconómico familiar en el éxito o fracaso académico (Majoribanks, 1996; Glik, \& White, 2004; Gil Flores, 2013; Escudero Muñoz, 2016; Santos Guerra, \& De la Rosa Moreno, 2016), ni la constatación de que las posibilidades de ascenso social son bajas para los sectores desfavorecidos, debido entre otras razones a la mella que hace en ellos el fracaso escolar (Gimeno Sacristán, 2013). Pero como contrapunto, también encontramos estudios que resaltan cómo las relaciones afectivas entre progenitores e hijos pueden minimizar los efectos negativos del bajo nivel económico y social de las familias, ya que estas relaciones no sólo alcanzan especial importancia en aspectos personales y afectivos, sino que "influyen a su vez en el desarrollo integral del niño, en la adquisición del sentido de competencia y en su comprensión del mundo" (Yin, \& Han, 2008, p. 209).

Por otro lado, es altamente conocida la gran influencia de las percepciones de los docentes sobre los y las estudiantes en cuanto a los resultados académicos y expec- 
tativas de futuro (Rosenthal, \& Jacobson, 1968; Goicochea, 2008; Ballestín González, 2015; García Vargas, 2015). Ahora bien, cuando hablamos de alumnado inmigrante, a esta perspectiva hay que sumarle las preconcepciones culturales y relacionales del profesorado que, en ocasiones, no comparte o no llega a comprender las especificidades de la cultura de origen de sus estudiantes:

...el estereotipo puede provocar lo que se conoce como efecto Pigmalión, es decir, al identificar a los individuos con una serie de características los estigmatizados tienden a actuar conforme a dicho perfil que se les atribuye. Cuando el estereotipo, como es habitual, implica atribuciones negativas, este suele conducir a un pobre rendimiento académico y a una anulación de las expectativas con que los inmigrantes suelen llegar al país de destino (Fuentes Gómez-Calcerrada, 2014, p. 66).

Si además tomamos en consideración el género, encontramos trabajos que evidencian cómo, a pesar de los aceptables resultados académicos de las jóvenes en la educación obligatoria, el profesorado mantiene bajas expectativas sobre sus posibilidades de acceso a la educación superior, atribuyendo esta percepción negativa a las culturas de los países de origen, fundamentalmente las de procedencia magrebí (Sirin, Ryce, \& Mir, 2009; Fernández Sierra, 2017).

Junto a ello, la institución educativa, como organización pedagógico-curricular, juega un papel esencial en la construcción de las expectativas académicas de las estudiantes (Wells, 2010; Escobedo Peiro, Sales Ciges, \& Traver Martí, en prensa), mostrando cómo la estructura organizativa y relacional de las escuelas y el currículum tradicional por disciplinas, distancian la acción pedagógica de las necesidades sociales multiculturales, promoviendo un saber descontextualizado que no ayuda en la construcción de expectativas de éxito académico, sobre todo cuando los referentes culturales son diferentes, lejanos e, incluso, antagónicos al hegemónico de acogida.

A pesar de ello, la visión de la educación como elemento de promoción social y de aumento de posibilidades personales y sociolaborales futuras sigue siendo un discurso muy asentado en nuestra sociedad y, en esa línea, las familias inmigrantes, especialmente las madres, ven en la escuela una excelente oportunidad para el porvenir de su prole, depositando gran parte de sus esperanzas en lo que pueda ofrecerles el sistema educativo, generándoles altas aspiraciones (Dusi, 2007) y convirtiéndose en el elemento medular en la construcción de las expectativas académicas de las y los jóvenes (Feliciano, \& Rubent, 2005; Llorent, Llorent-Bedmar \& Mata-Justo, 2015), resaltando su importancia y repercusión en la promoción y mejora social, cultural y económica de sus vástagos, siendo éste, para muchas de ellas, el objetivo primordial del proceso migratorio.

En este aspecto, en investigaciones anteriores (Fernández Sierra, 2013; 2017), habíamos constatado la emergencia de la figura materna como incitadora positiva en la participación y continuidad de las jóvenes en el sistema educativo; por lo que decidimos seguir indagando de qué manera inciden las madres y su contexto en la superación de la situación de desventaja y abandono, relacionándola con las prácticas pedagógicas cotidianas y las creencias asentadas en la comunidad educativa. En esta línea de análisis, 
el objetivo de la investigación que aquí presentamos ha sido acercarnos a las jóvenes inmigrantes en el contexto escolar para estudiar la construcción de expectativas que se crean, reflexionando sobre cómo éstas perciben su paso e inclusión en la escuela y qué perspectivas de futuro van elaborando en relación a las oportunidades formativas que éste les proporciona, intentando interrelacionar la influencia que prácticas docentes, familias, y relaciones con los pares ejercen sobre ello.

\section{Método}

Presentamos doce historias singulares de vida socioeducativa de niñas inmigrantes -cinco europeas del este/siete magrebíes; seis de primaria/seis de ESO-, con la finalidad de explicar y comprender cómo elaboran sus expectativas y cómo sus contextos familiares y socioculturales, y el sistema educativo y sus profesionales, influyen, limitan o posibilitan la consecución de sus deseos y perspectivas de futuro socioeducativo. Los criterios para seleccionarlas fueron: participantes de los Estudios de Caso (Stake, 2005) realizados en una investigación matriz en tres aulas de sexto de Primaria y tres de primero de ESO de centros públicos almerienses con más del 10\% de alumnado inmigrante -lo que permitió contextualizar el nicho ecológico escolar-, que fueran chicas, con idioma materno distinto al español, que nacieran y vivieran en un país extranjero, y que se incorporasen al sistema educativo español a partir del segundo ciclo de Primaria. Se negoció el acceso al campo con los docentes, y la participación con las jóvenes y con sus familias.

Los instrumentos utilizados fueron: entrevistas en profundidad y semiestructuradas elaboradas en el seno del equipo investigador, validando su idoneidad y lenguaje, con expertos y con jóvenes inmigrantes, y permitiendo flexibilidad a los investigadores para recabar información emergente de relevancia singular de cada joven. Se realizaron entre ocho/doce a cada una de las estudiantes -indagando en sus percepciones, recuerdos, vivencias y sensaciones sobre la vida estudiantil pasada, presente y aspiraciones futuras-, entre dos/tres a cada madre -profundizando en su visión sobre sus hijas-, y de cinco/siete a sus diferentes profesores/as -incidiendo en sus expectativas y sus prácticas pedagógicas con las alumnas inmigrantes -; siendo grabadas en audio, transcritas y negociadas. Éstas fueron contextualizadas por los datos obtenidos contemporáneamente a través de la observación participante dos días a la semana durante dos semestres, en aulas, espacios comunes y recreos, incorporando la descripción de relaciones y acción pedagógica en el cuaderno de campo/investigador.

El proceso de análisis y categorización para la elaboración de las narrativas -abordadas desde la información que las jóvenes han ido proporcionando en las sucesivas entrevistas, contrastada y complementada en polifonía con las aportaciones de sus madres y docentes, y triangulada con las descripciones del contexto escolar-, ha sido el siguiente: a lo largo de la recogida de información y a través de reuniones periódicasquincenales del equipo investigador, se fueron exponiendo los temas emergentes de cada historia, propiciando la elaboración de categorías comunes a la investigación y singulares de cada biografía, promoviendo la vuelta al campo para recabar información. Con los datos, cada investigador armó el relato singular poniendo el índice en categorías emergentes de especial relevancia para la investigación (unidades de 
análisis), sin obviar la idiosincrasia propia de cada estudiante (unidades biográficas); posteriormente, se negociaron los significados de las voces presentes en dichos relatos a través de unidades de codificación por extractos discursivos, con cada uno de las y los informantes. Construidas las historias singulares, el equipo investigador transversalizó las categorías en un relato cruzado (Pujadas Muñoz, 1992) elaborado en base a los análisis que aquí se presentan.

La codificación para la lectura es: (Ha pseudónimo:x), ubicando el primer término el dato en el relato singular y aclarando el segundo la persona que lo aporta: estudiante (pseudónimo), madre (madre), docente (prof) o investigadores (invest).

\section{Resultados}

Para la exposición de las evidencias que han emergido de la investigación, hemos seguido un proceso de categorización y triangulación que ha cristalizado en cinco apartados que utilizamos como guía de análisis: a) el sostén e impulso materno-filial en la construcción de expectativas; b) el papel que juega el ambiente socio-familiar y cultural; c) la incidencia de las relaciones con los pares en los centros educativos; d) las concepciones de los docentes sobre el alumnado inmigrante; e) la repercusión de la "vieja" pedagogía.

\section{Tándem madre-hija: la fuerza de la complicidad}

El elemento principal de referencia e influencia en la construcción de las expectativas de futuro de las niñas inmigrantes son sus madres. Éstas intentan planificar la educación y otros aspectos de la vida social de sus hijas con la intención primordial de protegerlas, fomentando su integración en la sociedad de acogida, llegando en algunos casos a evitar o reducir el contacto de las jóvenes con el pasado o con su país de origen:

Cuanto menos relación tengamos con lo que dejamos allí, más fácil será para Aniusca [...] Es importante para nosotras que ella sienta este país como su patria. No quiero crear lazos artificiales con mi país, que dificulten su integración aquí... ( $\mathrm{H}^{\mathrm{a}}$ Aniusca: madre).

Si analizamos la percepción que las chicas tienen sobre su realidad, se observa cómo suelen coincidir a menudo con sus madres en el sentimiento y en las manifestaciones de querer distanciarse de su pasado, aunque sus razonamientos son de índole diferente, más personales y muy ligados a su experiencia migratoria y de desarraigo del lugar de procedencia. Sofía nos explica cómo, cuando salió de Rumanía, su único deseo era ver a su madre que había hecho el proceso emigratorio dos años antes:

[...] no pensaba en nada más -escarba en sus recuerdos-, pero ahora veo que mi futuro está aquí... me gustaría hacer una carrera. No quiero volver porque allí no podría hacer una carrera, porque vale mucho dinero..., además, allí ya no conozco a nadie y no quiero volver a empezar [...], yo ya me siento española... (Haㅗofía: Sofía). 
La segunda gran aspiración de dichas madres es favorecer que sus hijas consigan lo que ellas piensan que les conviene a medio y largo plazo (Franzé Mudaró, 2008; Intxausti, Etxeberria, \& Joaristi, 2014). En este sentido y a pesar de las duras condiciones de trabajo que a menudo soportan, son las madres las que claramente sostienen y planifican el futuro de sus hijas. Deseos como el que manifiesta la madre de Dorina, de procedencia rumana, respecto a su hija de 12 años que cursa sexto de Primaria, afirmando con seguridad:

Pienso que mi hija podría ser una buena abogada, me gustaría abogada o médico [...] Yo una abogada lo veo muy bien, es un trabajo muy decente, puede conocer mucha gente, conocer sus problemas y resolverlos, ayudarle... $\mathrm{y}$ vivir bien con ese trabajo ( $\mathrm{H}^{\mathrm{a}}$ Dorina: madre).

Estas determinaciones maternas contagian a las hijas por empatía o por convencimiento; así, Ilmia, buena estudiante, escolarizada en otro centro también en el último curso de Educación Primaria, hija de una mujer lituana, razona:

[...] me preocupa ir al Instituto, porque las cosas quizás sean diferentes... más tarde iré a estudiar algo más importante [...] iré a la Universidad, mi madre lo dice..., ella quiere dejar el invernadero, trabajar en un almacén y después llegar a dependienta de una tienda... y para mí, la Universidad (Hallmia: Ilmia).

Para analizar esta estrecha relación madres-hijas, acudimos a tres circunstancias concurrentes: En primer lugar, resaltar el hecho de que la mayoría de estas madres han vivido el proceso migratorio sin el acompañamiento físico ni simbólico del padre biológico de la hija; habiéndonos sorprendido la proporción de mujeres que han manifestado a lo largo de la investigación, directa o sutilmente, que han sido problemas con su pareja lo que ha desencadenado su proceso migratorio:

Cuenta -la madre- que los motivos de su emigración fueron fundamentalmente la ruptura de pareja a partir de los malos tratos recibidos y el asedio posterior que recibía [...] No quería que su hija estuviese bajo la custodia de su padre por entender que no era una persona muy equilibrada [...] (HậSofía: invest).

En segundo lugar, hay que enfatizar la madurez socio-personal de estas niñas y la asunción de su responsabilidad en el presente y futuro del tandem hija-madre, como se pone de manifiesto en el cuidado de los hermanos o el doble trabajo que desempeñan en el colegio y en el hogar. Así lo resalta la maestra: "A Nadira le pasa como a otras muchas, trabaja doble jornada, escolar y en casa" (Hâa Nadira: prof). En tercer lugar, destacar la necesidad personal que tienen estas adolescentes de encontrar cobijo y amparo en ese único miembro estable y accesible de la familia, cuando todo lo demás le es en mayor o menor grado extraño, hostil o, al menos, no amigable. 


\section{Tejido sociocultural y familiar: condicionante destacado}

El ambiente socio-familiar en el que se desenvuelven las chicas -entendido como el sistema de relaciones que la adolescente y su familia establecen entre sí y ambos con la sociedad de acogida-, es otro factor esencial en la creación de sus perspectivas de futuro. La presión socio-escolar se hace más evidente y visible si a la actitud suspicaz y más o menos machista de los contextos de acogida, le sumamos la pertenencia a un contexto cultural discriminatorio respecto a las mujeres en sus geografías de origen; lo que unido al miedo o los recelos de algunas familias a perder la capacidad de protección o control de sus hijas, produce en éstas efectos contrapuestos que dificultan su incorporación inclusiva al contexto de acogida. Significativas son en este aspecto las palabras de Haima, magrebí estudiante de ESO de 15 años:

Mi madre no nos deja que vayamos con la gente de aquí [...] dice que no nos vayamos a buscar problemas. Tiene miedo a que nos echemos un novio de aquí - ¿Y te gustaría volver a tu país?- Sí, pero de vacaciones, yo prefiero que mis hermanos mayores se vengan... ( $\mathrm{H}^{\mathrm{a}}$ Haima: Haima).

Esta dualidad de apego a sus orígenes y familia, junto a la atracción de unos modos de vida que desea compartir con sus compañeras de la sociedad de acogida, provocan contradicciones y aspiraciones encontradas en la construcción de su futuro personal, sociocultural y profesional. En esta línea, la influencia del pensamiento feminista y de la igualdad de género -no sólo de la sociedad de acogida sino también de sus contextos de origen (Mirza, 2009; Huang, 2013)-, ha calado en muchas de estas chicas, revelándose, aun levemente, si la familia actúa de forma diferente con las hijas que con los hijos en algunas cuestiones escolares; así lo expresa Fatema -15 años- cuando no le permiten ir a un viaje de estudios, frente a su hermano de 14 años que sí consigue el permiso familiar: “[...] eso pasa porque soy una chica... es injusto" (Hâatema: Fatema).

No obstante, hemos de ser prudentes con todo tipo de generalización de los modos de actuar y de reaccionar, ya que en ello concurren variedad de factores y circunstancias. En esa línea, hallamos ejemplos o casos que contradicen las representaciones sociales y profesionales sobre ciertas culturas, especialmente cuando pensamos en familias musulmanas. Es el caso de Naima y Salma, ambas de 15 años y de procedencia magrebí, que internalizan de manera distinta sus vivencias migratorias. La primera asistió en su país de nacimiento a una escuela urbana mixta, antes de incorporarse al sistema educativo andaluz donde lleva seis años; su padre había emigrado previamente, y tras progresar económicamente, se produjo la reagrupación familiar. En el marco escolar, Naima encuentra diferenciaciones respecto a su país de origen:

Yo voy con mis amigas al parque... Yo salgo con amigas, alguna española... Con niñas de mi edad... Mi padre dice que parezco un niño [...], aquí es igual, me trata igual. En Marruecos se dice que las niñas no se tienen que juntar con los niños, pero aquí no, aquí yo me puedo ir con los niños [...] Mi padre dice que estudie lo que quiera, mis tíos me dicen que medicina o 
abogada pero a mí me gusta policía, desde chiquitita... En Marruecos, aunque estudies mucho, hay que viajar lejos (para ir a la Universidad) y a lo mejor no te dejan, pero aquí sí, aquí la universidad está al lado y puedes viajar a Madrid, en Marruecos eso es muy difícil. (Hânaima: Naima).

Por el contrario, Salma, hija de una familia extensa relativamente acomodada, con mujeres ejerciendo trabajos liberales (una tía médica y otra veterinaria), en contra de lo que en principio cabría esperar por su estatus sociofamiliar, manifiesta resistencias externas e intrapsíquicas ante su inclusión en el nuevo contexto sociocultural. Sin duda hay factores y aspectos sutiles, menos evidentes, que influyen en estas actitudes y recreación de expectativas, especialmente las experiencias personales relacionadas con las vivencias e internalización del proceso migratorio:

A mí no me gusta salir. Mi madre dice: sal, vete, no te quedes aquí; pero a mí no me gusta. Mi madre me regaña, me dice vete con tus amigas a estudiar, pero es que a mí no me gusta ir [...] La gente no nos quiere toda igual... Piensan que en Marruecos todo es malo. Yo lo pasé mal [al venir], allí tenía muchas amigas y mucha familia y al llegar aquí y no encontrar a nadie pues me he sentido... no sé, muy mal (Hª̂Salma: Salma).

\section{Compañeros y compañeras de clase: relaciones fluctuantes}

Explica Espósito (2011) que "las interacciones sociales que los estudiantes desarrollan con los pares en las instituciones escolares -y en las aulas- proporcionan un espacio importante de análisis acerca de la creación de la identidad personal" (p.88). En concordancia con esta evidencia, en nuestro estudio, el tercer elemento considerado ha sido la influencia que ejercen los sistemas relacionales que establecen estas jóvenes con sus compañeros y compañeras de colegio en la construcción de sus expectativas.

Lo primero que evidenciamos es la connivencia, en los mismos grupos-clase e individuos, del binomio aceptación-rechazo circunstanciales de los niños y niñas inmigrantes por sus pares. $\mathrm{O}$ sea, que en las edades y niveles educativos en los que estamos llevando a cabo este trabajo, no podemos decir que se detecten claramente jóvenes racistas, xenófobos o todo lo contrario, sino que más bien hemos constatado situaciones, momentos y circunstancias en los que los mismos individuos y/o grupos de preadolescentes reaccionan de manera contrapuesta. Significativo en este aspecto es el caso de Anastasia, de origen ruso, que, como la investigadora va describiendo en su cuaderno de campo, ha alcanzado altas cotas de participación, aceptación e interrelación con sus compañeros y compañeras:

Anastasia es una niña que baila y canta muy bien [...] lleva tres curso en el centro y, aunque el primer año tuvo dificultades, ahora está muy integrada [...] es una líder. Las compañeras hacen lo que les propone, participa en los recreos con niñas de otras clases y hay buen ambiente entre niños y niñas (Haㅡastasia: invest). 
Sin embargo, ante un hecho ocasional y violento ajeno a la escuela, en el que se vieron implicados algunos inmigrantes, se provocó una grave reacción racista en el contexto social de la ciudad donde habita. El peso de la xenofobia y del machismo del ambiente social autóctono, penetró en el centro educativo haciendo especial mella sobre las chicas inmigrantes, como ella. En ese momento, la investigadora escribe:

Este día se ha presentado Anastasia llorando y sumamente afectada en el despacho de la directora, le faltaba el habla... Un grupo de niños la había espetado en el patio: ¡Tu madre es una puta que viene a quitarle el dinero a los hombres de aquí! ( $\mathrm{H}^{\mathrm{a}}$ Anastasia: invest).

Estas conductas, social y personalmente graves, son relevantes pedagógica y didácticamente porque nos indican que se palpa un racismo o xenofobia subyacente en estos estudiantes, fruto en gran parte del contagio social, que aunque en muchos casos no sean actitudes e ideologías totalmente asentadas sino circunstanciales, se hace necesaria una acción educativa que encauce dichas concepciones y pautas conductuales agresivas. Ponen en evidencia la importancia de estar atentos y basar las acciones docentes en cuestiones profundas, para ayudarles a superar esta presión social y reconducir a los y las jóvenes por el camino inverso a las manifestaciones de tinte xenófobo que surgen o se provocan intencionadamente en su entorno, cebándose doblemente contra las adolescentes.

\section{Creencias docentes: limitaciones y rebaja de expectativas}

El cuarto elemento tomado en consideración para el análisis de la construcción de expectativas de las chicas, son sus profesores y profesoras, puesto que existe correlación entre las expectativas de aquellos sobre sus estudiantes y el éxito escolar de éstos. Con respecto a las niñas inmigrantes, no sólo se repite esta constante, sino que se evidencian peculiaridades y matices más sutiles, relacionados con prejuicios, concepciones seudoculturales y estereotipos sobre las posibilidades académicas y sociales futuras que los docentes se formulan respecto a ellas, evidenciándose una especie de "comprensión paternalista" que nos da pistas sobre dichas expectativas:

[...] realmente, el profesorado es más permisivo con las faltas de asistencia de las niñas...; de alguna manera entiende los problemas y necesidades de las familias para atender a los hermanos pequeños, ayudar en casa...; ya se sabe que las mujeres asumen más tareas en estos asuntos [...] es una pena, pero está como bastante asumido en general ( $\mathrm{H}^{\mathrm{a}}$ Zaida: prof).

Concepciones que no podemos considerar aisladas o esporádicas, sino evidencias de la prevalencia de un pensamiento profesional hegemónico sobre el fenómeno de la inmigración y de las expectativas de los y las docentes respecto a las perspectivas de futuro de estas jóvenes en razón de su origen geográfico-cultural, con afirmaciones como las siguientes: "Aquí tenemos la cultura A, la B y la C. La A, la de los autóctonos; la $\mathrm{B}$, la de los magrebíes; la $\mathrm{C}$, la de las demás nacionalidades..." (Hạa Fatema: prof); 
o "Las chicas marroquíes, la mayoría tienen ya novio buscado y sus expectativas es casarse y quedarse en su casa. Otras por suerte están cambiando, las que llevan aquí más tiempo y los padres cambian la mentalidad" (Hânaima: prof).

Ahora bien, aun partiendo de esa manifestación determinista de la cultura y del aprendizaje, el profesorado actúa de forma muy diferente en sus clases en razón de su pensamiento pedagógico, ideología sociocultural y compromiso profesional. Hemos observado cómo algunos docentes consideran una complicación y contrariedad la incorporación de este alumnado a los centros de enseñanza, porque -aseveran- les supone "disrupción de sus clases" (HậSofía: prof), argumentando que no pueden seguir los niveles, que no les entienden y que perjudican a los demás. Procuran que estén fuera de su aula, delegando la tarea educativa en las clases de apoyo o en las de atención lingüística el mayor tiempo posible, o bien, los mantienen pasivos realizando tareas nimias para que no "interrumpan" (HNadira: prof). Actitudes que preocupan a algunos compañeros: "Ante la llegada de estos alumnos al centro no se planteó nada [...], incluso algunos compañeros, cuando hablaban de ellos, les llamaban moros..., tenían claro que iban a pasar del tema [...]" (HâSalma: prof).

En contraposición, otros profesionales interpretan el fenómeno de la inmigración como una situación socioeducativa a la que el sistema de enseñanza ha de dar respuesta desde una visión intercultural, respetuosa con la diversidad, que aporta riqueza cultural y nuevos retos educativos. Son profesionales que intentan innovar y renovar la escuela, no sólo para los y las hijas de inmigrantes, sino para adecuarla a las nuevas necesidades personales y sociales: "Somos los profesores los que tenemos que coger el toro por los cuernos. Lo que sí sería interesante es el intercambio de experiencias..." (Hâlma: prof).

No obstante, estos docentes, bien considerados en sus centros, se encuentran con dificultades para implicar a sus colegas en experiencias pedagógicas innovadoras, especialmente en ESO, con alta formación disciplinar en sus áreas de conocimiento pero de insuficiente formación pedagógica y didáctica. Cualquier reto educativo lo perciben como un problema de instrucción sobre contenidos disciplinares de su especialidad: "Para mí los alumnos inmigrantes no suponen ningún problema, siempre que tengan superado el idioma. Una vez superado esto no hay diferencias [...] el que quiere estudia y el que no, no, como los de aquí" (HāDorina: prof).

Actitudes y actuaciones pasivas que se han manifestado más evidentes en el caso de las niñas inmigrantes, ya que éstas suelen adoptar posturas pacientes y silenciosas en las clases; invisibilizándose y dedicándose preferentemente a la realización de los trabajos que les encarga la maestra de atención lingüística o a realizar las fichas que les proporcionan en las clases de apoyo: "Sí hay diferencias entre niños o niñas (inmigrantes), ellas son más pacíficas... ellos respetan menos las normas [...] Nairet es muy lenta, pero buena, calladita... Ella va haciendo tranquilamente los trabajos que trae de la clase de español“ ( $\mathrm{H}^{\mathrm{a}}$ Nairet: prof).

\section{Envejecimiento de la pedagogía: el pecado del sistema}

En opinión de Touraine (2005), la crisis de la escuela se debe al envejecimiento de la pedagogía y a la posición atemporal de la institución escolar en la que se ha apos- 
tado por continuar con la formación del capital humano de otras épocas. Sin duda, la incorporación del alumnado inmigrante al sistema educativo ha evidenciado más claramente este desfase pedagógico al obviar que el pensamiento relativista de un mundo globalizado y diverso, demanda de una escuela incitadora que favorezca en sus estudiantes una construcción de identidad inclusiva. En este sentido, en nuestro estudio hemos constatado cómo las rutinas asentadas en las aulas y en los centros escolares, dificultan sobremanera la incorporación inclusiva y la comunicación intercultural de la comunidad educativa. Así lo explica el coordinador del proyecto de interculturalidad:

[...] se convocó un primer encuentro de experiencias de iniciativas interculturales [...] nuestra intención era ver qué se estaba haciendo en la práctica bajo ese rótulo [...] Las experiencias que allí se presentaron como innovadoras en educación intercultural, eran básicamente enseñar castellano a niños inmigrantes..., ni trabajo en la escuela, ni proyectos, ni trabajo en organización... (Hªniusca: prof).

Las prácticas pedagógicas hegemónicas observadas pivotan en torno a tres rutinas didácticas que limitan las posibilidades de ofrecer alternativas, haciéndose más ineficaces e insostenibles conforme avanzamos hacia una sociedad más multicultural y diversa, al tiempo que va aumentando el número de hijos e hijas de inmigrantes en nuestras escuelas.

La primera de esas rutinas es el enfoque homogeneizador impersonal de la acción docente, unido a una concepción unidireccional e individualista de los procesos de enseñanza-aprendizaje que no garantiza la comunicación, el intercambio, la aproximación, el conocimiento y el reconocimiento del otro. Cuando se dice querer adecuar las enseñanzas a las singularidades, se hace desde un instrumentalismo academicista que limita aún más la adaptación personal: "ella nunca molesta... ahí la tengo haciendo fichas... ¡Que voy a hacer con ella en clase de Lengua si no domina el idioma!" (Hânairet: prof). Sistemas de trabajo individualistas, que no personalizados, que no favorecen la comunicación intercultural, aislando sobremanera a las chicas que tienden a centrarse en clase en tareas individuales y a agruparse en el recreo con un reducido grupo de ellas, también inmigrantes, con poca posibilidad de interrelación con oriundas o grupos de jóvenes más amplios y variados.

La segunda rutina es de carácter organizativo-didáctico. Se sigue gestionando el tiempo de aprendizaje de cada estudiante en función de una concepción de déficit individual en cuanto a la acumulación de contenidos disciplinares; por lo tanto, la estrategia pedagógica hegemónica es la multiplicación de intervenciones reparadoras fuera del aula ordinaria en horario escolar (ATAL, Mediación, Recuperación, Orientación, etc.). Sistemas de apoyo extra-aula, con los que se pretende ayudar a los niños y niñas en el aprendizaje de algunos conocimientos disciplinares básicos, que a la postre descuelgan a los estudiantes inmigrantes de sus grupos, dificultándoles sobremanera su incorporación y participación en clase. Este modo de organizar la compensación educativa que potencia la creación de grupos cerrados, desubicados y güetizados, dificulta la comunicación intercultural, estigmatizando al que va a ellos, impulsándoles 
hacia el aislamiento, especialmente a las chicas que llegan a sentirse más cómodas y seguras en los momentos de separación del grupo-clase, asumiendo incluso el lenguaje discriminatorio con normalidad:

Me gusta más estar aquí (clase de ATAL). Estoy mejor en la clase de los moros que con los otros niños [...] en la clase de los españoles me da vergüenza..., como los españoles saben más que nosotros, si no decimos bien una palabra, se ríen ( $\mathrm{H}^{\mathrm{a}}$ Nadira: Nadira).

La clave que cierra el arco de estas rutinas pedagógicas es el sistema de evaluacióncalificación, basado en la comprobación de rendimientos sobre contenidos disciplinares eminentemente memorísticos. La enseñanza basada en la retentiva y reproducción de las síntesis de los libros de texto o apuntes, además de pedagógicamente desfasada y poco útil para todo el alumnado, supone un esfuerzo extra para las jóvenes inmigrantes -impidiéndoles dedicar su tiempo escolar a adquirir aprendizajes significativos y relevantes-, colaborando a su expulsión del sistema -aún más que a los oriundos- y desalentando sus expectativas académicas y de futuro:

[...] los españoles sólo lo leen unas pocas veces y pueden escribir con sus palabras, mientras que nosotras tenemos que estudiar más para poder escribir exactamente bien las respuestas [...] Tengo buena memoria, pero sólo si uso mucho tiempo estudiando para memorizar ( $\mathrm{H}^{\mathrm{a}}$ Zaida: Zaida).

\section{Conclusiones}

Desde el paradigma cualitativo, en el que hemos ubicado este trabajo, no buscamos generalizaciones axiomáticas, sino presentar una descripción y análisis de la realidad que permita al lector construir su propia transferibilidad (Guba, 1989). En este sentido, consideramos importante finalizar con una serie de ideas fuerza emergidas de la investigación, a modo de conclusiones sobre las que seguir reflexionando.

La figura de las madres, mayoritariamente trabajadoras en la agricultura intensiva y limpieza doméstica, ha impulsado y contagiado a sus hijas con sus propios anhelos y perspectivas de futuro, manifestándose como uno de los ejes fundamentales en la construcción de expectativas de estas chicas. Hemos podido observar una simbiosis emocional madre-hija, en el que miran al futuro intentando soltar amarras identitarias y culturales, con la finalidad de superar el dolor de la adaptación en soledad, el desarraigo y el no querer volver a pasar nuevamente por el desapego sociocultural generado por el hecho migratorio; pues, cómo otras investigación han constatado (Ramos Tovar, 2009; Briones Pérez, 2010; Gómez García, 2013), las personas migradas, en especial los y las adolescentes, quedan marcadas en su identidad por ese proceso al vivenciar situaciones de desapego cultural, dificultad en la comunicación, falta de apoyo emocional-social, y el traslado a un espacio desconocido, en ocasiones hostil, que les impulsa a un intento de mimesis en el nuevo espacio vital.

Más allá de la relación madre-hija y especialmente en el contexto de las familias musulmanas, en las que a priori el choque cultural parecería mayor ante el triángulo 
migración-género-expectativas socioculturales y académicas, nos hemos encontrado con sentimientos contradictorios en las jóvenes a la hora de afrontar lo que las familias esperan culturalmente de ellas y lo que ellas desean, tanto en cuanto a su cultura de procedencia como a la de acogida, escenificada a menudo en "el espejo" de sus compañeras de colegio. Esta dicotomía, del ser-con-los-otros, les lleva a responder bajo una lógica personal que nada tiene que ver con patrones estandarizados, encontrando jóvenes que buscan en sus raíces culturales musulmanas la forma de relacionarse con el mundo, a la vez que viven en un contexto familiar aculturizado, social y económicamente en la sociedad de acogida.

Por otro lado, las relaciones con los grupos de pares -esenciales en la construcción de la identidad de las jóvenes estudiantes por cuanto el éxito en la relaciones sociales incidirán en la construcción de su imagen y la percepción de sí mismas en el futuro (Read, 2011; McInerney, 2014)- encallan en un maleable binomio aceptación-rechazo, ejerciendo una violencia simbólica latente, no tanto por actitudes o ideologías asentadas, como por situaciones puntuales del contexto social que desembocan en conductas manifiestamente xenófobas que, en el caso de las jóvenes inmigrantes, se potencian con prejuicios machistas de carácter étnico, cultural y de género, lo que en parte concuerda con el Estudio del Movimiento contra la Intolerancia (2007) al señalar que casi uno de cada tres estudiantes entre 13 y 15 años muestran actitudes racistas.

En lo pedagógico, la mayoría de las prácticas imperantes en los centros estudiados continúan ancladas en un corsé disciplinar, etnocéntrico, reparador, asimilacionista e invisibilizador de otras culturas y de lo femenino (Blanco García, 2011; Fernández Sierra, 2011; Torres Santomé, 2011; González-Peiteado, \& Pino-Juste, 2016; Jiménez Delgado, 2016), casi reduciendo la intervención compensatoria a la asimilación lingüística como mágica panacea del éxito académico y relacional de estas jóvenes. La introducción de variables culturales heterogéneas, directamente observables en las aulas, han hecho más evidentes y visibles las contradicciones y limitaciones del currículo técnico predominante y de la metodología eminentemente transmisiva tanto de contenidos como de valores hegemónicos; sin embargo no han tenido la fuerza de reorientar el paradigma pedagógico, prevaleciendo currículas no diseñados para abordar la diversidad cultural y de género desde la inclusividad, sino más bien elaborados desde la homogeneización y estandarización. El profesorado, en gran parte, ha mostrado tener asumida una concepción intrapsíquica respecto a la responsabilidad de los procesos de aprendizaje del sujeto migrado pensando que son estos chicos y chicas quienes han de adecuarse a la realidad sociocultural de acogida, fagocitándose en la cultura hegemónica.

En esa línea, aun considerando la actuación de la Administración andaluza incorporando diversos profesionales para atender al alumnado inmigrante y el desarrollo de programas interculturales fuera y dentro del ámbito educativo, éstos no han logrado una transformación en las estrategias organizativas y docentes que permita una escolaridad inclusiva de estas estudiantes, por cuanto el espíritu y principios de dichos programas no ha sido provocar una reformulación de las orientaciones pedagógicas hegemónicas tecnificadas poco favorecedores de la integración, más allá de sus habilidades personales para adaptarse a la nueva realidad socioescolar. Esto, unido a las políticas educativas de rendimiento, que han fracasado 'parcialmente' en su función compensatoria (Pérez Gómez, 2012; Escudero Muñoz, 2016), y a los contextos xenófobos y machistas aún pre- 
sentes en ciertos sectores sociales, a menudo producen desamparo educativo en cuanto a la potenciación de confianza y de perspectivas de futuro, reflejados sobre manera en las niñas inmigrantes, ya que ellas carecen de otros referentes, apoyos o recursos que puedan aportarles lo que la escuela no les ofrezca en este terreno.

Como ya advertía Francis (2010, p. 480) al manifestar que "las instituciones educativas, como las escuelas, han influido tradicionalmente en la construcción social de la personalidad, reproduciendo estereotipos de género, perpetuando rutinas e influyendo en los pensamientos de los adolescente durante muchos años", hemos observado cómo algunos docentes escenifican de manera sutil, prejuicios culturales y estereotipos que acotan las posibilidades académicas de las estudiantes en base a sus países de origen, siendo las niñas magrebíes las que reciben menos aliento en lo que a expectativas futuras proyectan estos docentes. No obstante, en sentido contrario, la profesionalidad y la visión de la escuela como lugar de construcción holística de la persona, acerca a otros docentes a sensibilizarse con estas estudiantes, incorporando una cercanía empática con ellas, especialmente las maestras, y llevando a cabo prácticas pedagógicas innovadoras que parten de la construcción de un nuevo escenario inclusivo, en el que la singularidad multicultural y de género no se ven obviados.

Para finalizar, y considerando la singularidad a la hora de interpretar y sentir estas ideas-fuerza por parte de cada una de las chicas que han compartido sus vivencias con nosotros, observamos cómo, aún con sensaciones de inestabilidad o pérdida ante el choque cultural y socioeducativo, consiguen -contra el pronóstico socioprofesional mayoritario-, reedificarse e ir construyendo sus ilusiones y expectativas de futuro conforme se mantienen en el sistema de enseñanza y progresan en él.

\section{Referencias}

Ballestin González, B. (2015). De "su cultura es muy fuerte" a "no se adapta a la escuela": alumnado de origen inmigrante, evaluación y efecto Pigmalión en primaria. RASE, $8(3), 361-379$.

Blanco, N. (coord.). (2011). Educar en femenino y masculino. Madrid: Akal.

Briones Pérez, E. (2010). La aculturación de los adolescentes inmigrantes en España. Salamanca: Universidad de Salamanca.

Delors, J. (1996). La Educación encierra un tesoro. Informe de la Comisión internacional sobre la Educación para el Siglo XXI. Madrid: Santillana-UNESCO.

DeSeCo (2003). Definition and selection of competencies: Theoretical and conceptual foundation. Recuperado de: http://www.portal-stat.admin.ch/deseco/deseco_finalreport_summary.pdf

Dusi, P. (2007). Scuola e famiglia; un rapporto internazionale. Milano: Vita e pensiero.

Escobedo Peiro, P. Sales Ciges, A., \& Traver Martí, J. (en prensa). La voz del alumnado: su silencio y la cultura profesionalista. Educación XX1.

Escudero Muñoz, J.M. (2016). Inclusión y exclusión educativa: realidades, mejoras y propuestas. Valencia: Nau Llibres.

Esposito, J. (2011). Hill girls, consumption practices, power, and city style: raced and classed production of femininities in a higher education setting. Gender and Education, 23:1, 87-104 
Feliciano, C., \& Rumbaut, R. (2005). Gendered paths: Educational and occupational expectations and outcomes among adult children of immigrants. Ethnic and Racial Studies, 28(6), 1087-1118.

Fernández Sierra, J. (2011). Formar para la economía del conocimiento vs educar para la sociedad del conocimiento: una visión desde la pedagogía. Málaga: Aljibe.

Fernández Sierra, J. (coord.) (2013). Transitar la cultura: niños y niñas inmigrantes en la educación obligatoria. Malaga: Aljibe.

Fernández Sierra, J. (2017). Alumnado inmigrante en la ESO: Vulnerabilidad pedagógica del sistema educativo. Educación XX1, 20 (1), 121-140, doi: 10.5944/educXX1.12855

Francis, B. (2010). Re/theorising gender: female masculinity and male femininity in the classroom? Gender and Education, 22(5), 477-490.

Franzé Mudaró, A. (2008). Diversidad cultural en la escuela. Algunas contribuciones antropológicas. Revista de Educación, 345, 111-133.

Fuentes Gómez-Calcerrada, J. (2014). Identidad cultural en una sociedad plural: propuestas actuales y nuevas perspectivas. Bordón, 66(2), 61-74. DOI: 10.13042/ Bordon.2014.66204.

García Vargas, J. (2015). El efecto Pigmalión y su efecto transformador a través de las expectativas. Perspectivas docentes, 57, 40-43.

Gil Flores, J. (2013). Medición del nivel socioeconómico familiar en el alumnado de Educación Primaria. Revista de Educación, 362, 298-322. DOI: 10.4438/1988-592XRE-2011-362-162.

Gimeno Sacristán, J. (2013). En busca del sentido de la educación. Madrid: Morata.

Glick, J.E., \& White, M.J. (2004). Post-Secondary School Participation of Immigrant and Native Youth: The Role of Familial Resources and Educational Expectations. Social Science Research, 33, 272-299.

Goicochea, M. (2008). El adolescente inmigrado latinoamericano en la escuela española. Las miradas docentes. Revista Española de Educación Comparada, 14, 79-102.

Gómez García, R. (2013). La educación intercultural. Madrid: Ediciones Diez de Santos.

González-Peiteado, M., \& Pino-Juste, M. (2016). Los estilos de enseñanza: construyendo puentes para transitar las diferencias individuales del alumnado. Revista Complutense de Educación, 27(3), 1175- 1191. http://dx.doi.org/10.5209/rev_RCED.2016. v27.n3.47563

Guba, E.G. (1989). Criterios de credibilidad en la investigación naturalista. En J. Gimeno Sacristán, \& A. Pérez Gómez (Coords.) La enseñanza: su teoría y su práctica (pp. 148-165). Madrid: Akal.

Jiménez Delgado (2016). Discursos interculturales y prácticas asimilacionistas: algunas contradicciones en el sistema educativo español. Convergencia, 71, 41-66.

Huang, C. (2013). Gender differences in academic self-efficacy: a meta-analysis. European Journal of Psychology of Education, 28, 1-35.

INE. (2015). Anuario Estadístico de España 2015. Educación. Recuperado de http://www. ine.es/prodyser/pubweb/anuario15/anu15_03educa.pdf

Intxausti, N. Etxeberria, F., \& Joaristi, L. (2014). ¿Coinciden las expectativas escolares de la familia y del profesorado acerca del alumnado de origen inmigrante?. RELIEVE, 20 (1), DOI: 10.7203/relieve.20.1.3804. 
Llorent, V. Llorent-Bedmary, V., \& Mata-Justo, J. (2015). Expectativas académicas de los inmigrantes de segunda generación en Setúbal y Faro (Portugal). Revista de Pedagogía Social, 26, 315-336.

Majoribanks, K. (1996). Family socialization and children's school outcomes: An investigation of a parenting model. Educational Studies, 22(1), 3-11.

McInerney, D. (2014). Educational Psychology: Constructing Learning. Frenchs Forest: Parson.

Mirza, H. (2009). Race, Gender and Educational Desire: Why Black Women Succeed and Fail. USA: Routledge.

Movimiento contra la intolerancia (2007). Informe RAXEN 33. Recuperado de http:// www.movimientocontralaintolerancia.com/download/raxen/33/33Completo.pdf

Pérez Gómez, A. I. (2008). ¿Competencias o pensamiento práctico? La construcción de los significados de representación y de acción". En J. Gimeno (Ed.), Educar por competencias, ¿qué hay de nuevo? (pp. 59-102). Madrid: Morata.

Pérez Gómez, A. I. (2012). Educarse en la era digital. Madrid: Morata.

Pujadas Muñoz, J. (1992). El método biográfico: el uso de las historias de vida en ciencias sociales. Madrid: CIS

Ramos Tavor, M. (2009). Migración e identidad: emociones, familia, cultura. Monterrey, Mexico: Fondo Editorial de Nuevo León.

Read, B. (2011). Britney, Beyoncé, and me primary school girls' role models and constructions of the 'popular' girl. Gender and Education, 23, 1-13.

Rosenthal, R., \& Jacobson, L. (1968). Pygmalion in the classroom: Teacher expectations and pupils' intellectual development. New York, EEUU: Holt, Rinehart and Winston.

Santos Guerra, M.A. y De la Rosa Montero, L. (2016). La participación de las familias de alumnos y alumnas inmigrantes en las instituciones educativas. Revista Internacional de Formação de Professores (RIFP),1(1), p. 101-120

Sirin, S., Ryce, P., \& Mir, M. (2009). How teachers' values affect their evaluation of children of immigrants: Findings from Islamic and public schools. Early Childhood Research Quarterly, 24(4), 463-473.

Stake, R. E. (2005). Investigación con estudio de casos. Madrid: Morata.

Tejada Fernández, J., \& Ruiz Bueno, C. Evaluación de competencias profesionales en educación superior: retos e implicaciones. Educación XX1, 19(1), 17-38.

Torres Santomé, J. (2011). La justicia curricular. El caballo de Troya de la cultura escolar. Madrid: Morata.

Touraine, A. (2005). Un nuevo paradigma para comprender el mundo de hoy. Barcelona: Paidós.

Wells, R. (2010). Children of immigrants and educational expectations: The roles of school composition. Teachers College Record, 112(6), 1679-1704.

Yin, Y., \& Han, M. (2008). Parental Contributions to Southeast Asian American Adolescents' Well-Being. Youth E Society, 40(2), 289-306.

Fecha de recepción: 27 de abril de 2016

Fecha de revisión: 27 de abril de 2016

Fecha de aceptación: 14 de noviembre de 2016 